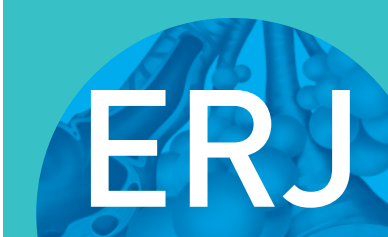

open research

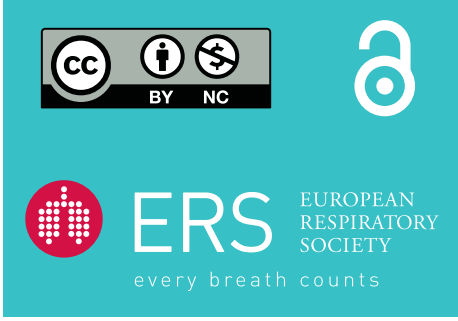

\section{Seeing the world through different lenses: activity registration differs between two validated accelerometers}

To the Editor:

Encouragement of regular physical activity is a cornerstone in the treatment of COPD [1], since physical inactivity leads to increased mortality and hospitalisation rates [2,3]. Additionally, regular physical activity has shown a protective effect on lung function decline in subgroups [4].

Accurate measurement of the regular physical activity level is key for setting up patient-tailored physical activity coaching programmes and the assessment of interventions. Wearable devices are frequently used to assess physical activity levels and, in daily practice, triaxial monitors are the most reliable devices to measure physical activity. The PROactive consortium showed that the Actigraph GT3X (Actigraph LLC, Pensacola, FL, USA) and DynaPort MoveMonitor (McRoberts BV, The Hague, the Netherlands) are the most valid accelerometers to estimate energy expenditure and discriminate between different walking speeds in patients with COPD [5, 6]. Although strong correlations for both accelerometers with activity-related energy expenditure (AEE) have been shown, data are lacking with regard to time spent in physical activity or postures, such as walking and sitting.

Currently, the effects of pulmonary rehabilitation in patients with severe COPD who undergo bronchoscopic lung volume reduction with endobronchial valves are studied (SoLVE study; NCT03474471). Prior to the start of the SoLVE study, we noticed disproportional high non-worn times in our Actigraph measurements. To select the most appropriate device for our study, we aimed to investigate whether or not there were differences in non-worn time and type of activity registration between the Actigraph GT3X and Dynaport MoveMonitor in the first 10 participants of our study. The SoLVE study was approved by our local medical ethics committee and all patients signed informed consent.

Participants wore both accelerometers simultaneously for seven consecutive days and were instructed to register the non-worn time in a diary. The Actigraph GT3X was worn on the right waist and the DynaPort MoveMonitor on the lower back according to their instructions for use. In one patient, the Actigraph GT3X measurement could not be analysed due to technical problems. Therefore, analyses were performed on the results of nine patients (age: $64 \mathrm{yrs}$, male: $55.6 \%$, forced expiratory volume in $1 \mathrm{~s}: 28 \%$ ). One patient took off both accelerometers overnight because of sleeping problems and therefore has a substantial lower lying time and higher non-worn time. Wilcoxon signed rank tests were performed to measure differences between both accelerometers. Two-way mixed intraclass correlation coefficients were calculated to determine consistency between the Actigraph GT3X and DynaPort MoveMonitor measurements. A comparison of the results of both accelerometers is given in table 1.

In comparison with the DynaPort MoveMonitor, the Actigraph GT3X registered significantly lower lying time and AEE, and significantly higher standing and non-worn time. The DynaPort MoveMonitor derived information about activities such as shuffling, walking, stair walking and cycling, whereas locomotion time is not specifically registered by the Actigraph GT3X.

@ERSpublications

Although accelerometers are extensively validated to estimate energy expenditure in patients with COPD, they show significant differences in non-worn time and type of activity registration http://bit.ly/32fa7WR

Cite this article as: van der Molen MC, Hartman JE, Vanfleteren LEGW, et al. Seeing the world through different lenses: activity registration differs between two validated accelerometers. ERJ Open Res 2020; 6: 00262-2019 [https://doi.org/10.1183/23120541.00262-2019].

Copyright $\odot$ ERS 2020. This article is open access and distributed under the terms of the Creative Commons Attribution NonCommercial Licence 4.0. 


\begin{tabular}{|c|c|c|c|c|}
\hline & Actigraph GT3X & DynaPort MoveMonitor & Median difference & p-value \\
\hline Steps per day & $1761(787-2905)$ & $2446(821-3914)$ & $421(-517-1183)$ & 0.110 \\
\hline $\begin{array}{l}\text { Activity-related energy } \\
\text { expenditure per day kcal }\end{array}$ & $86(43-139)$ & $424(357-704)$ & $356(279-579)$ & 0.008 \\
\hline Lying time $\%$ & $10.6(1.3-37.7)$ & $39.7(6.0-58.0)$ & $30.3(4.7-39.4)$ & 0.008 \\
\hline Sitting time \% & $44.1(17.4-54.1)$ & $43.7(36.8-51.0)$ & $0.4(-5.1-19.4)$ & 0.594 \\
\hline Standing time $\%$ & $11.6(4.1-30.8)$ & $7.2(3.0-18.0)$ & $3.6(-1.2-17.3)$ & 0.015 \\
\hline Locomotion time \% & & $3.2(1.1-6.1)$ & $1.0(-2.8-13.2)$ & 0.214 \\
\hline Non-worn time \% & $32.1(10.7-44.8)$ & $0.4(0-40.7)$ & $30.7(1.7-43.7)$ & 0.008 \\
\hline Non-worn time diary \% & $0.3(0-41.3)^{\#}$ & $0.3(0-41.3)$ & & \\
\hline
\end{tabular}

Although the Actigraph GT3X registered a significantly higher standing time in comparison with the DynaPort MoveMonitor, there was no significant difference between the Actigraph GT3X standing time and the sum of the Dynaport MoveMonitor standing and locomotion time. It can therefore be hypothesised that the Actigraph GT3X registers locomotion time as standing time.

The Actigraph GT3X registered a lower number of steps per day than the Dynaport MoveMonitor, but this was not statistically significant and the intraclass correlation coefficients were good between both measurements (absolute $=0.76$, consistency $=0.80$ ). A Bland-Altman plot was in agreement with these findings.

The AEE was significantly different between both devices and the intraclass correlation coefficients were poor (absolute $=0.35$, consistency $=0.35$ ). The Bland-Altman plot was in agreement with these findings. The lower AEE estimate of the Actigraph GT3X could possibly be explained by the high non-worn time. The Actigraph GT3X registered non-worn time was significantly higher than the diary registered non-worn time, whereas there was no significant difference between the diary and the by DynaPort MoveMonitor registered non-worn time. Given the disproportionally low lying time (10.6\%) registered by the Actigraph GT3X, it seems reasonable to assume that most lying time is registered as non-worn time. However, non-worn time was also often registered throughout the day, even after correction of registration conflicts.

In conclusion, we found that two validated accelerometers showed significant differences in non-worn time and type of activity registration in our small sample of patients with COPD. The technical field in medicine is fast evolving and we tend to rely on validated instruments. Accelerometers are gaining importance in research, clinical practice, and disease and health management. Although both accelerometers are extensively validated against doubly labelled water [5], we recommend that the choice of accelerometer should be based on the physical activity outcome of interest. Based on our data, we decided to use the DynaPort MoveMonitor for our study given our interest in daily physical activity rather than energy expenditure and the concerning high non-worn times as registered by the ActiGraph GT3X. However, further research is warranted to validate type of activity registration of triaxial accelerometers in patients with COPD.

Marieke C. van der Molen ${ }^{1}$, Jorine E. Hartman ${ }^{1}$, Lowie E.G.W. Vanfleteren $\odot^{2}$ and Dirk-Jan Slebos ${ }^{1}$

${ }^{1}$ Dept of Pulmonary Diseases, University of Groningen, University Medical Center Groningen, Groningen, The Netherlands. ${ }^{2}$ COPD Center, Sahlgrenska Universitetssjukhuset, Gothenburg, Sweden.

Correspondence: Marieke C. van der Molen, Pulmonary Diseases, University Medical Centre Groningen Thoraxcentre, Groningen, Netherlands. E-mail: m.c.van.der.molen@umcg.nl

Received: 23 Sept 2019 | Accepted after revision: 3 Oct 2019

Support statement: This study was supported by Lung Foundation Netherlands grant: 5.1.17.171.0. Funding information for this article has been deposited with the Crossref Funder Registry.

Conflict of interest: M.C. van der Molen has nothing to disclose. J.E. Hartman has nothing to disclose. L.E.G.W Vanfleteren reports grants, personal fees and nonfinancial support from Pulmonx, personal fees and nonfinancial support from Menarini, grants and personal fees from Astrazeneca, personal fees from Chiesi, GSK, Novartis and Boehringer, and grants and nonfinancial support from Fisher \& Paykel, outside the submitted work. D-J. Slebos has nothing to disclose. 
References

1 Vogelmeier CF, Criner GJ, Martinez FJ, et al. Global strategy for the diagnosis, management, and prevention of chronic obstructive lung disease 2017 report. Am J Respir Crit Care Med 2017; 195: 557-582.

2 Garcia-Aymerich J, Lange P, Benet M, et al. Regular physical activity reduces hospital admission and mortality in chronic obstructive pulmonary disease: A population based cohort study. Thorax 2006; 61: 772-778.

3 Waschki B, Kirsten A, Holz O, et al. Physical activity is the strongest predictor of all-cause mortality in patients with COPD: A prospective cohort study. Chest 2011; 140: 331-342.

4 Garcia-Aymerich J, Lange $\mathrm{P}$, Benet $\mathrm{M}$, et al. Regular physical activity modifies smoking-related lung function decline and reduces risk of chronic obstructive pulmonary disease: A population-based cohort study. Am J Respir Crit Care Med 2007; 175: 458-463.

5 Rabinovich RA, Louvaris Z, Raste Y, et al. Validity of physical activity monitors during daily life in patients with COPD. Eur Respir J 2013; 42: 1205-1215.

6 van Remoortel H, Raste Y, Louvaris Z, et al. Validity of six activity monitors in chronic obstructive pulmonary disease: A comparison with indirect calorimetry. PLoS One 2012; 7: e39198. 